\title{
Multi-Walled Carbon Nanotubes (MWCNTs) Activate Apoptotic Pathway Through ER Stress: Does Surface Chemistry Matter?
}

This article was published in the following Dove Press journal: International Journal of Nanomedicine

\author{
Yongbing Sun' \\ Jianping Gong' \\ $\mathrm{Yi} \mathrm{Cao}{ }^{2}$
}

\begin{abstract}
'National Engineering Research Center for Solid Preparation Technology of Chinese Medicines, Jiangxi University of Traditional Chinese Medicines, Jiangxi, Nanchang 330006, People's Republic of China; ${ }^{2}$ Key Laboratory of EnvironmentFriendly Chemistry and Application of Ministry of Education, Laboratory of Biochemistry, College of Chemistry, Xiangtan University, Xiangtan 4III 05, People's Republic of China
\end{abstract}

Purpose: Physicochemical properties play a crucial role in determining the toxicity of multi-walled carbon nanotubes (MWCNTs). Recently we found that MWCNTs with longer length and smaller diameters could induce toxicity to human umbilical vein endothelial cells (HUVECs) through the activation of endoplasmic reticulum (ER) stress. In this study, we further investigated the possible contribution of hydroxylation and carboxylation to the cytotoxicity of MWCNTs.

Methods: The HUVECs were exposed to pristine (code XFM19), hydroxylated (code XFM20; content of hydroxyl groups $1.76 \mathrm{wt} \%$ ) and carboxylated (code XFM21; content of carboxyl groups $1.23 \mathrm{wt} \%$ ) MWCNTs, respectively. Then, the internalization, cytotoxicity, oxidative stress and activation of apoptosis-ER stress pathway were measured.

Results: In consequence, all types of MWCNTs could be internalized into the HUVECs, and the cellular viability was significantly reduced to a similar level. Moreover, the MWCNTs increased intracellular reactive oxygen species (ROS) and decreased glutathione (GSH) to similar levels, indicating their capacity of inducing oxidative stress. The Western blot results showed that all types of MWCNTs reduced BCL-2 and increased caspase-3, caspase-8, cleaved caspase- 3 and cleaved caspase- 8 . The expression of ER stress gene DNA damageinducible transcript 3 (DDIT3) and protein level of chop were only significantly induced by XFM20 and XFM21, whereas protein level of p-chop was promoted by XFM19 and XFM21. In addition, the pro-survival gene $X B P-1 s$ was significantly down-regulated by all types of MWCNTs.

Conclusion: These results suggested that MWCNTs could induce cytotoxicity to HUVECs via the induction of oxidative stress and apoptosis-ER stress, whereas a low degree of hydroxylation or carboxylation did not affect the toxicity of MWCNTs to HUVECs.

Keywords: multi-walled carbon nanotubes, MWCNTs, human umbilical vein endothelial cells, HUVECs, endoplasmic reticulum (ER) stress, hydroxylation, carboxylation, ER stress

\section{Introduction}

Multi-walled carbon nanotubes (MWCNTs) represent tubular structures with unique mechanical, physical and electrical properties, which render them be popular in commercial products, such as batteries, sporting goods and supercapacitors. ${ }^{1-3}$ In recent years, the potential applications of MWCNTs in biomedical areas have also gained extensive research interests. For example, MWCNTs could be used to separate pharmaceutics. ${ }^{4}$ Moreover, MWCNTs also show great potential as novel nanocarriers for cancer therapy. ${ }^{5}$ Certain types of MWCNTs possess antibacterial properties and
Correspondence: Jianping Gong National Engineering Research Center for Solid Preparation Technology of Chinese Medicines, Jiangxi University of Traditional Chinese Medicines, Jiangxi, Nanchang 330006, People's Republic of China Tel +86-079l-87II9623

Email gongjianping64@I63.com 
might be used as novel antibacterial materials. ${ }^{6}$ However, the biocompatibility of MWCNTs should be taken into consideration to ensure the safe uses of MWCNTs. Particularly, there is an urgent need to assess the biocompatibility of MWCNTs to human endothelial cells, due to the potential damage of MWCNTs towards human blood vessels. ${ }^{7,8}$

It is well accepted that the physicochemical properties of nanomaterials (NMs) could significantly influence their interactions with biological systems, and understanding the impact of physicochemical factors on biocompatibility of NMs is crucial to design bio-safe NMs. ${ }^{9,10}$ For the cases of MWCNTs, the diameters, lengths and surface chemistry are crucial physicochemical characteristics determining their biocompatibility. ${ }^{11}$ Recently, we found that MWCNTs with longer lengths and shorter diameters were more toxic to human umbilical vein endothelial cells (HUVECs) due to the activation of ER stress, ${ }^{12,13}$ which suggested that length and diameter are important parameters for the rational design of biocompatible MWCNTs. However, the possible influence of surface chemistry on the toxicity of MWCNTs to HUVECs has not yet been investigated. Previous studies focused on the biocompatibility of MWCNTs with different surface chemistry to other cell types rather than endothelial cells. For example, Hamilton et al found that the increase of the carboxylation degree of MWCNTs decreased their bioactivity both in vivo and in vitro. ${ }^{14}$ In another study, Liu et al showed that carboxylation of MWCNTs increased the biocompatibility of MWCNTs to L02 cells, suggesting the application prospect of carboxylated MWCNTs for biomedical uses. ${ }^{15}$ However, De Marchi et al recently found that the functionalized MWCNTs were generally more toxic to Ruditapes philippinarum compared with non-functionalized MWCNTs at the same mass concentrations. ${ }^{16}$ To the best of our knowledge, only one study compared the toxicity of MWCNTs with different surface chemistry to HUVECs, and showed that carboxylation of MWCNTs increased the toxicity of MWCNTs to HUVECs. ${ }^{17}$ However, further investigation is needed to explore the influence of surface chemistry on the toxicity of MWCNTs to endothelial cells.

Therefore, the present study compared the toxicity of pristine MWCNTs, hydroxylated MWCNTs and carboxylated MWCNTs to HUVECs, respectively. This study used HUVECs as the in vitro model because HUVECs have been commonly used to investigate the toxicity of NMs to human endothelial cells. ${ }^{7}$

\section{Materials and Methods \\ The Overall Study Design}

Three types of commercially available MWCNTs, namely pristine MWCNTs (code XFM19), hydroxylated MWCNTs (code XFM20) and carboxylated MWCNTs (code XFM21), were characterized by multiple technologies, including field emission scanning electron microscope (FE-SEM), transmission electron microscope (TEM), dynamic light scattering (DLS) and Fourier transform infrared spectroscopy (FTIR). HUVECs were exposed to various concentrations of MWCNTs for $24 \mathrm{hrs}$. After exposure, cytotoxicity was measured by cell counting kit- 8 (CCK-8) assay. The internalization of MWCNTs into HUVECs was studied by transmission electron microscope (TEM) and side-scattering light (SSC). The intracellular reactive oxygen species (ROS) and glutathione (GSH) were measured to detect the oxidative stress, which has been found to be involved in the toxic effects of MWCNTs. ${ }^{18}$ To further investigate the molecular mechanism associated with the toxicity of different types of MWCNTs to HUVECs, the biomarkers associated with ER stress were measured. It has been previously shown that NM exposure could induce ER stress of cells, which consequently leads to the activation of apoptosis pathway. ${ }^{19}$ Meanwhile, NM-induced oxidative stress might also lead to prolonged ER stress. ${ }^{19,20}$ For MWCNTs, we recently showed that MWCNTs could activate ER stress in HUVECs, which is related with the lengths ${ }^{12}$ and diameters ${ }^{13}$ of MWCNTs. However, the possible influence of surface chemistry has not been investigated. Herein, we measured the mRNA levels of DNA damage-inducible transcript 3 (DDIT3; also known as chop, C/EBP homologous protein) and spliced $\mathrm{X}$-box binding protein $1(X B P-1 s)$ by qRT-PCR, and protein levels of p-chop, chop, BCL-2, caspase-3 and inositolrequiring enzyme 1 (IRE1 $\alpha$ ) by Western blot.

\section{Cell Culture}

HUVECs were purchased from ScienCell Research Laboratories (Carlsbad, CA, USA). The cells were cultured in endothelial cell medium (ECM) according to the protocol from ScienCell Research Laboratories.

\section{Characterization of MWCNTs}

Pristine MWCNTs (code XFM19), hydroxylated MWCNTs (code XFM20) and carboxylated MWCNTs (code XFM21) were purchased from Nanjing XFNANO Materials Tech Co., Ltd. According to the supplier, the content of hydroxyl groups in XFM20 has been determined as $1.76 \mathrm{wt} \%$, and the 
content of carboxyl groups in XFM21 has been determined as $1.23 \mathrm{wt} \%$ by using X-ray photoelectron spectroscopy. However, further increasing the degree of hydroxylation/ carboxylation is difficult due to the diameters of XFM20. In this study, all types of MWCNTs were characterized using FE-SEM (SU-5000, Hitachi, Japan) as well as TEM (JEM1400 Plus, JEOL, Japan). The diameters and lengths were measured from 20 randomly selected MWCNTs in each sample by using ImageJ software. To confirm the presence of hydroxyl or carboxyl groups, the Fourier Transform infrared spectroscopy (FTIR) spectrum was recorded using Nicolet iS50R FTIR Spectrometer (Thermo Scientific ${ }^{\mathrm{TM}}$, USA). To prepare the suspensions of MWCNTs, MWCNTs were dispersed in $2 \%$ fetal bovine serum (FBS) at the concentration of $1.28 \mathrm{mg} / \mathrm{mL}$ under sonication for $16 \mathrm{mins}$ by using the ultrasonic processor FS-250N (Shanghai Shengxi, China). After sonication, the MWCNTs were immediately diluted by cell culture medium to desired concentrations for exposure. The control cells were incubated with the same amount of vehicles. To measure hydrodynamic size, zeta potential and polydispersity index (PDI), the MWCNT suspensions were prepared as $32 \mu \mathrm{g} / \mathrm{mL}$ in water or cell culture medium and then analyzed by using Zetasizer nano ZS90 (Malvern, UK).

\section{CCK-8 Assay}

CCK-8 assay was used to indicate the changes of cellular viability, especially the decrease of mitochondrial activities. Briefly, HUVECs were seeded at the density of $4 \times 10^{4} /$ well in 24-well plates and grown for 2 days. After that, the cells were exposed to various concentrations of XFM19, XFM20 and XFM21 for $24 \mathrm{hrs}$. After exposure, the cells were thoroughly rinsed once by Hanks solution, and CCK-8 assay was done by using commercial kits purchased from Beyotime Biotechnology (Nantong, China). The data for CCK-8 measurement were summarized in Table S1.

\section{TEM Observation of MWCNT-Exposed HUVECs}

TEM was used to investigate the internalization of MWCNTs. Briefly, HUVECs were seeded at $5 \times 10^{5}$ on $60 \mathrm{~mm}$ diameter cell culture Petri dishes and grown for 2 days before exposure. The cells were incubated with cell culture medium (control) or $64 \mu \mathrm{g} / \mathrm{mL}$ XFM19, XFM20 and XFM21. After $3 \mathrm{hrs}$ exposure, the cells were rinsed and then scratched by using a cell scraper. After centrifuge, the cells were fixed with $2.5 \%$ glutaraldehyde in PBS overnight, post-fixed with $1 \% \mathrm{OsO}_{4}$ for $3 \mathrm{hrs}$, dehydrated in a graded series of ethanol and embedded in epoxy Resin (Epon 812). The samples were then sectioned using an ultramicrotome at $70 \mathrm{~nm}$, placed on carbon film supported by copper grids, stained with uranyl acetate and lead citrate, and observed under a TEM (JEM-1230, JEOL Ltd., Tokyo, Japan) operated at $80 \mathrm{kV}$.

\section{Light Scatter Analysis}

HUVECs were cultured in 12-well plates at a density of $1 \times 10^{5}$ cells/well and grown for 2 days before exposure to $64 \mu \mathrm{g} / \mathrm{mL}$ of XFM19, XFM20 or XFM21 for 24 hrs. HUVECs incubated with cell culture medium for $24 \mathrm{hrs}$ were used as control. After exposure, the cells were removed from plates by using trypsin, and the changes in SSC were measured by using flow cytometry (BD LSRFortessa ${ }^{\mathrm{TM}}$, Franklin Lakes, NJ, USA).

\section{Intracellular ROS and GSH}

Intracellular ROS and GSH were measured by fluorescent probes 2',7'-dichlorofluorescin diacetate (DCFH-DA) and monochlorobimane (MCB; DCFH-DA and MCB were purchased from Sigma-Aldrich), respectively. These endpoints were measured because they are sensitive to reflect oxidative status of stressed cells. For the assays, HUVECs were seeded in 96-well black plates at a density of $1 \times 10^{4} /$ well, grown for 2 days, and then exposed to various concentrations of MWCNTs for $24 \mathrm{hrs}$. After exposure, the cells were rinsed once, and stained by $10 \mu \mathrm{M}$ DCFH-DA or $50 \mu \mathrm{M} \mathrm{MCB}$ for about 30 mins. After rinsed once again, the fluorescence was read at excitation $360 \pm 44 \mathrm{~nm}$ and emission $460 \pm 40 \mathrm{~nm}$ (for GSH) or excitation $485 \pm 20$ $\mathrm{nm}$ and emission $528 \pm 20 \mathrm{~nm}$ (for ROS) by an ELISA reader (Synergy HT, BioTek, USA). The data for intracellular ROS and GSH measurement were summarized in Table S1.

\section{qRT-PCR}

The mRNA levels of ER stress genes DDIT-3 and XBP-1s as well as internal control glyceraldehyde-3-phosphate dehydrogenase $(G A P D H)$ were determined by qRT-PCR. Briefly, $2 \times 10^{5}$ per well HUVECs were seeded in 6-well plates and grown for 2 days before exposure to $64 \mu \mathrm{g} / \mathrm{mL}$ XFM19, XFM20 or XFM21 for 24 hrs. The cells incubated with cell culture medium were used as control. After exposure, total mRNA was extracted by using TRI Reagent ${ }^{\mathbb{R}}$ (Sigma-Aldrich, USA). cDNA was synthesized by using HiFiScript cDNA Synthesis Kit (Cwbiotech, Beijing, China), and qRT-PCR was done by using UltraSYBR 
Mixture (Cwbiotech, Beijing, China) on PikoReal ${ }^{\mathrm{TM}} \mathrm{qPCR}$ system (Thermo-Fisher, USA). The experiments were done following manufacturers' instructions. The primers used in this study are: GAPDH (NM_002046.7) forward (F-) primer ACAGCCTCAAGATCATCAGC, and reverse (R-) primer GGTCATGAGTCCTTCCACGAT (product length104 bp); DDIT3 (NM_001195057.1) F-primer GGAAACAGAGTG GTCATTCCC, and R-primer GGAAACAGAGTGGTCAT TCCC (product length 116 bp); XBP-1s (NM_001079539.1) F-primer CCGCAGCAGGTGCAGG, and R-primer GAGT CAATACCGCCAGAATCCA (product length $70 \mathrm{bp}$ ). Table S3 summarized the conditions for the qPCR amplification procedure. The mRNA levels were calculated by Livak method and expressed as the ratio between the mRNA level of the target genes and the internal control gene. The data for qRT-PCR are summarized in Table S2.

\section{Western Blot}

The protein levels of chop, p-chop, caspase-3, caspase-8, IRE $\alpha$ and BCL-2 were determined by Western blot. Briefly, $2 \times 10^{5}$ per well HUVECs were seeded on 6-well plates and grown for 2 days before exposure to $0 \mu \mathrm{g} / \mathrm{mL}$ (control) or 64 $\mu \mathrm{g} / \mathrm{mL}$ MWCNTs for $24 \mathrm{hrs}$. After exposure, the cells were rinsed twice by Hanks solution, and proteins were extracted by using RIPA lysis buffer with the presence of proteases inhibitor cocktail and PhosStop ${ }^{\mathrm{TM}}$ phosphatase inhibitor (Roche Diagnostics). After placed on ice for 10 mins, the supernatants were collected by 15 mins centrifuge at 12 ,$000 \mathrm{rpm}, 4^{\circ} \mathrm{C}$. The protein concentrations were measured by BCA method, and $50 \mu \mathrm{g} /$ sample proteins were mixed with loading buffer and then resolved on SDS-PAGE. The samples were transferred to a nitrocellulose membrane, blocked in non-fat milk for $1.5 \mathrm{hrs}$ at room temperature, and then incubated overnight at $4{ }^{\circ} \mathrm{C}$ with the primary antibody (1:500 p-chop rabbit antibody, Abcam, UK; 1:800 chop rabbit antibody, Proteintech, USA; 1:800 IRE1 $\alpha$ rabbit antibody, Proteintech, USA; 1:600 caspase-3 rabbit antibody, Proteintech, USA; 1:1000 caspase-8 rabbit antibody, Proteintech, USA; 1:1000 BCL-2 rabbit antibody, Proteintech, USA; $\alpha$-actin mouse antibody, Proteintech, USA). The blots were washed in $0.1 \% \mathrm{w} / \mathrm{v}$ Tween-PBS and then incubated with 1:5000 HRP goat anti-rabbit IgG (Proteintech, USA) for $1.5 \mathrm{hrs}$. After that, the blots were detected by SuperECL Plus chemiluminescence (Thermo pierce, USA). The data for Western blot are summarized in Table S2, and the unedited WB images are shown in Figure S1. The density of each band was determined by using ImageJ (NIH).

\section{Statistics}

Data are expressed as means \pm SD of means of three independent experiments ( $\mathrm{n}=3$ for each). For the data of CCK-8, ROS and GSH measurement, two-way ANOVA followed by Tukey HSD test was used to analyze the influence of concentrations of MWCNTs and surface chemistry on the toxicological effects. For the data of qRT-PCR and Western blot, one-way ANOVA was used to compare the differences, since only one concentration was used for these experiments.

\section{Results}

\section{Characteristics of MWCNTs}

In this study, multiple methods were used to characterize the MWCNTs. Both SEM images (Figure 1A) and TEM images (Figure 1B) indicated that all the samples contained bundles of MWCNTs even after sonication. The average diameters were calculated as $28.97 \pm 6.05 \mathrm{~nm}$ (XFM19), $30.46 \pm 11.63 \mathrm{~nm}$ (XFM20) and $31.03 \pm 5.37 \mathrm{~nm}$ (XFM21), and the average lengths were calculated as $1181.14 \pm 352.89 \mathrm{~nm}$ (XFM19), $1323.94 \pm 1025.13 \mathrm{~nm}($ XFM20) and $1256.59 \pm 454.73 \mathrm{~nm}$ (XFM21), respectively. The results from DLS measurement showed that all types of MWCNTs had similar hydrodynamic size, zeta potential and PDI in both water and cell culture medium (Figure 1C and D \& Table 1). It should be noticed that for non-spherical NMs like MWCNTs, DLS reported radius of a hypothetical hard sphere, which can make the hydrodynamic sizes different from their primary size. ${ }^{21}$ Meanwhile, the agglomerate/aggregate status could also influence the reported hydrodynamic size of MWCNTs.

The FTIR spectrum (Figure 2) exhibited the characteristic adsorption bands at $2930 \mathrm{~cm}^{-1}$ (C-H stretching) and $3450 \mathrm{~cm}^{-1}$ (-OH stretching) in the sample of XFM20, as well as $1640 \mathrm{~cm}^{-1}\left(\mathrm{C}=\mathrm{O}\right.$ stretching) and $3450 \mathrm{~cm}^{-1}(-\mathrm{OH}$ stretching) in the sample of XFM21, which confirmed that XFM20 and XFM21 were surface functionalized. ${ }^{22}$

\section{Cytotoxicity of MWCNTs to HUVECs}

The cytotoxicity of MWCNTs to HUVECs was investigated by CCK-8 assay and the result is shown in Figure 3. There was a modest but statistically significant decrease of cellular viability after exposure to $32 \mu \mathrm{g} / \mathrm{mL}$ or $64 \mu \mathrm{g} / \mathrm{mL}$ MWCNTs $(\mathrm{p}<0.01)$. However, ANOVA test indicated no difference among different types of MWCNTs $(p>0.05)$.

\section{The Internalization of MWCNTs}

As shown in TEM images, MWCNTs could be internalized into HUVECs (Figure 4A-H). Typically, we observed 

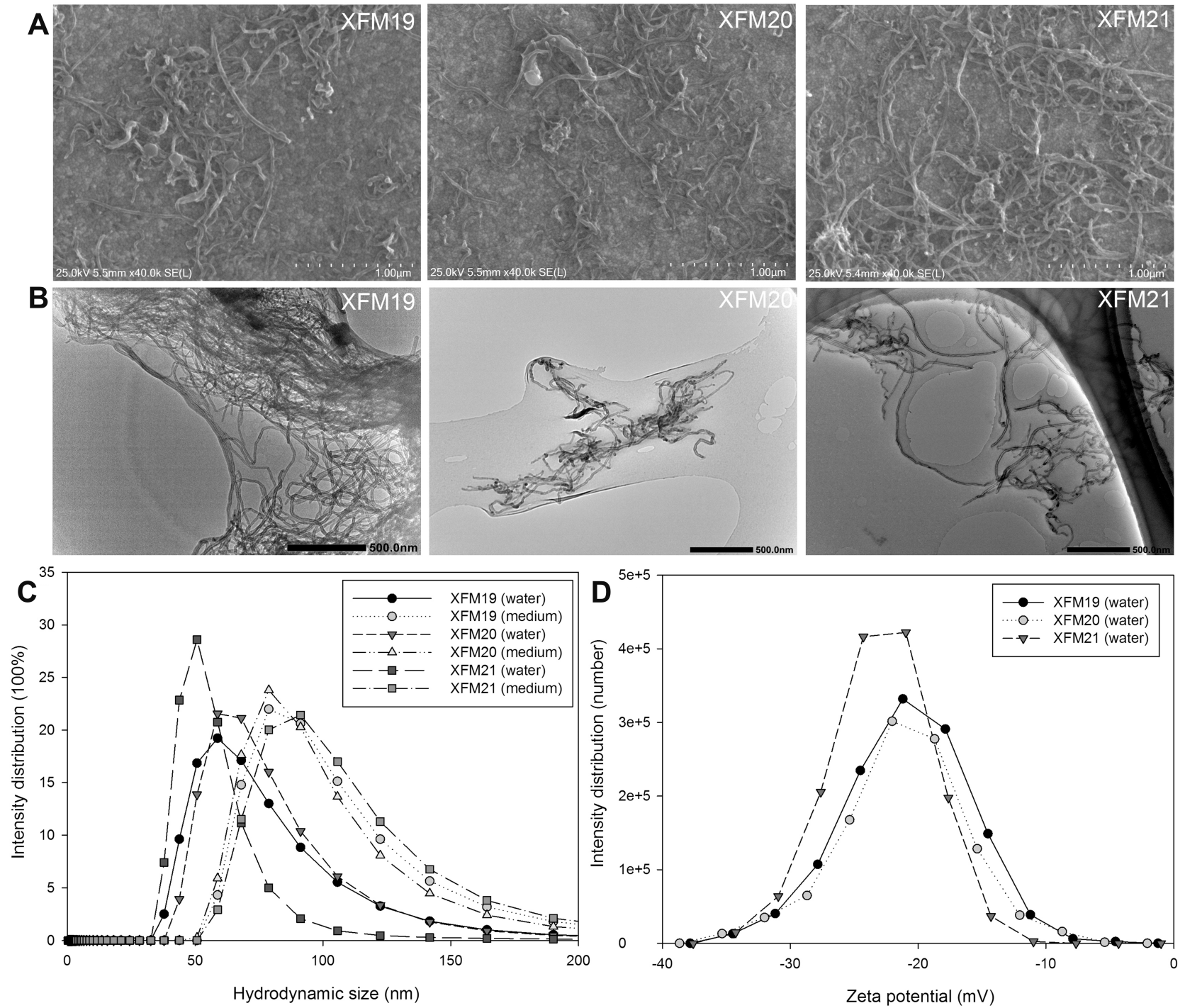

Figure I The characteristics of pristine MWCNTs (code XFMI9), carboxylated MWCNTs (code XFM2I) and hydroxylated MWCNTs (code XFM20). (A) SEM images; (B) TEM images; (C) the distribution of hydrodynamic size; and (D) the distribution of zeta potential.

MWCNTs near nuclei (labeled as N) and mitochondria (labeled as M). In addition, obvious mitochondrial swelling has been observed in MWCNT-exposed HUVECs compared with control. The results of light scattering showed that all types of MWCNTs increased SSC of HUVECs to a similar extent (Figure S2).

\section{Intracellular ROS and GSH}

The intracellular ROS and GSH were determined in HUVECs treated with MWCNTs. As shown in Figure 5, all types of MWCNTs significantly promoted intracellular ROS or decreased intracellular GSH at the concentrations of $8 \mu \mathrm{g} / \mathrm{mL}, 16 \mu \mathrm{g} / \mathrm{mL}, 32 \mu \mathrm{g} / \mathrm{mL}$ and $64 \mu \mathrm{g} / \mathrm{mL}(\mathrm{p}<0.01)$. Again, all the types of MWCNTs increased intracellular ROS or decreased intracellular GSH to a similar extent.

\section{The Activation of Apoptosis Pathway}

Because we observed that MWCNTs induced the most pronounced effects on cytotoxicity, ROS and GSH at the concentration of $64 \mu \mathrm{g} / \mathrm{mL}$, we used this concentration for the rest of experiments to investigate the mechanism. As shown in Figure 6, all types of MWCNTs significantly decreased the protein levels of BCL-2 $(\mathrm{p}<0.01)$, but exposure to XFM20 and XFM21 was associated with relatively lower levels of BCL-2 compared with the exposure of XFM19 $(\mathrm{p}<0.01$; Figure 6B). The protein level of caspase- 3 was only significantly induced by XFM19 $(\mathrm{p}<0.01)$, which is significantly higher than that in cells exposed to XFM20 or XFM21 $(p<0.01)$. The protein level of cleaved caspase-3 was significantly induced by all types of MWCNTs $(p<0.01)$, but 
Table I The Average Hydrodynamic Size, Zeta Potential and PDI of XFMI9, XFM20 and XFM2I

\begin{tabular}{|c|c|c|c|c|}
\hline $\begin{array}{l}\text { Types of } \\
\text { MWCNTs }\end{array}$ & Code & Hydrodynamic Size (nm) & Zeta Potential (mV) & PDI \\
\hline Pristine MWCNTs & XFMI9 & $\begin{array}{l}\text { I75.53 } \pm 1.44 \text { (water); } 210.73 \pm 3.80 \\
\text { (medium) }\end{array}$ & $\begin{array}{l}-20.13 \pm 0.67 \text { (water); }-1.54 \pm 3.5 I \\
\text { (medium) }\end{array}$ & $\begin{array}{l}0.250 \pm 0.029 \text { (water); } 0.276 \pm 0.004 \\
\text { (medium) }\end{array}$ \\
\hline $\begin{array}{l}\text { Hydroxylated } \\
\text { MWCNTs }\end{array}$ & XFM20 & $\begin{array}{l}\text { I74.20 } \pm 5.97 \text { (water); } 210.47 \pm 3.93 \\
\text { (medium) }\end{array}$ & $\begin{array}{l}-19.60 \pm 1.42 \text { (water); }-7.49 \pm 0.75 \\
\text { (medium) }\end{array}$ & $\begin{array}{l}0.257 \pm 0.007 \text { (water); } 0.27 I \pm 0.013 \\
\text { (medium) }\end{array}$ \\
\hline $\begin{array}{l}\text { Carboxylated } \\
\text { MWCNTs }\end{array}$ & XFM2I & $\begin{array}{l}\text { I87.30 } \pm 4.69 \text { (water); } 214.27 \pm 3.38 \\
\text { (medium) }\end{array}$ & $\begin{array}{l}-22.83 \pm 0.70 \text { (water); }-5.31 \pm 0.53 \\
\text { (medium) }\end{array}$ & $\begin{array}{l}0.268 \pm 0.019 \text { (water); } 0.294 \pm 0.026 \\
\text { (medium) }\end{array}$ \\
\hline
\end{tabular}

Abbreviations: MWCNTs, multi-walled carbon nanotubes; PDI, polydispersity index.

XFM20 induced significantly higher level of cleaved caspase-3 compared with that induced by XFM19 and XFM21 $(p<0.01$; Figure 6 C). The protein level of caspase- 8 was only significantly induced by XFM19 $(p<0.01)$, which is significantly higher than that in cells exposed to XFM20 $(\mathrm{p}<0.05)$ or XFM21 $(\mathrm{p}<0.01)$. The protein level of cleaved caspase- 8 was significantly induced by XFM20 and XFM21 $(\mathrm{p}<0.01)$, with XFM20 being more potent to induce cleaved caspase- 8 compared with XFM19 and XFM21 $(\mathrm{p}<0.01$; Figure 6D)

\section{Genes and Proteins Related with ER Stress}

As shown in Figure 7, the expression of DDIT3 was only significantly promoted by the exposure of XFM20 and XFM21 $(\mathrm{p}<0.01)$ but not XFM19 ( $>0.05)$. XFM20 induced significantly higher DDIT3 expression compared with that induced by XFM19 or XFM21 ( $<0.01$; Figure $7 A)$. The expression of $X B P-1 s$ was significantly down-regulated by

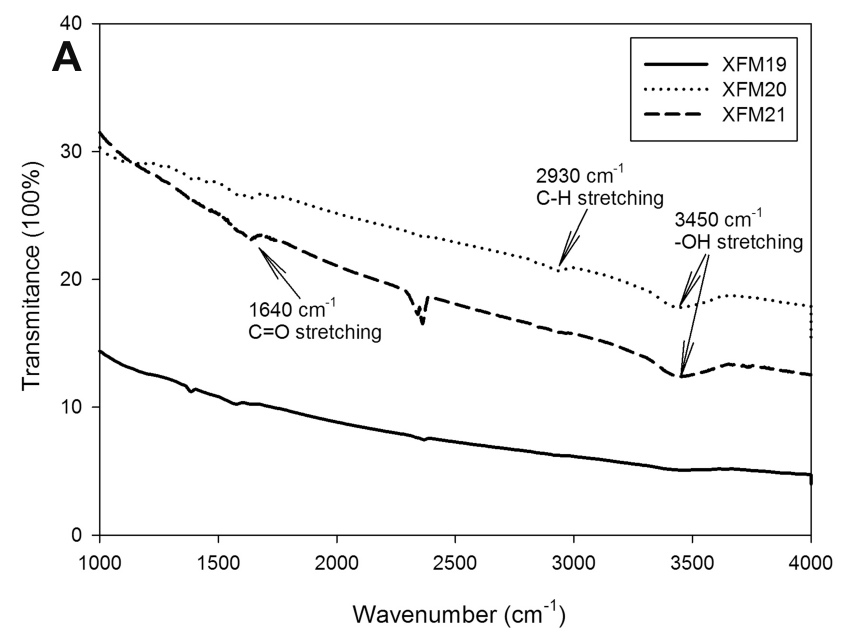

Figure 2 The FTIR spectrum of pristine MWCNTs (XFMI9), hydroxylated MWCNTs (XFM20) and carboxylated MWCNTs (XFM2I). all types of MWCNTs (Figure 7B). The protein level of IRE1 $\alpha$ was significantly enhanced by XFM19 and XFM20 exposure $(\mathrm{p}<0.01$ for both) but remained unaltered by XFM21 exposure ( $>0.05)$. XFM20 was the most potent to induce IRE1 $\alpha$ protein level, which is significantly higher compared with that induced by XFM19 and XFM21 exposure $(\mathrm{p}<0.01$; Figure 7D). The protein level of $\mathrm{p}$-chop was only modestly promoted by XFM19 $(\mathrm{p}<0.05)$ and XFM 21 $(\mathrm{p}<0.01)$, and XFM21 induced significantly higher $\mathrm{p}$-chop protein level than that induced by XFM19 $(\mathrm{p}<0.05)$ and XFM20 ( $<<0.01)$. The protein level of chop was markedly promoted by XFM20 and XFM21 ( $<0.01$ for both), and XFM21 was more effective than XFM19 and XFM20 to enhance the protein level of chop ( $\mathrm{p}<0.01$; Figure $7 \mathrm{E})$.

\section{Discussion}

In this study, we investigated the toxicity of pristine MWCNTs, hydroxylated MWCNTs and carboxylated MWCNTs to HUVECs through ER stress pathway. The cytotoxicity assay indicated that all types of MWCNTs significantly decreased cellular viability to a similar extent (Figure 3). Previous studies showed that the cytotoxicity of MWCNTs ${ }^{17}$ or single-walled carbon nanotubes ${ }^{23}$ to endothelial cells could be increased once they were carboxylated. Orecna et $\mathrm{al}^{17}$ further suggested that the cytotoxicity of carboxylated MWCNTs to human endothelial cells was due to the blockade of autophagic flux. However, we recently compared the cytotoxicity of XFM19 and XFM21 in HUVECs, alveolar-HUVEC co-culture, ${ }^{24}$ human aortic smooth muscle cells ${ }^{25}$ and HepG2 cells, ${ }^{26}$ and found that pristine and carboxylated MWCNTs induced cytotoxicity to similar extent in different in vitro cell models. In the present study, we found that the cytotoxicity of MWCNTs to HUVECs was not significantly influenced by hydroxylation or carboxylation on MWCNTs (Figure 3). Although the surface of MWCNTs was functionalized with hydroxyl or carboxyl 


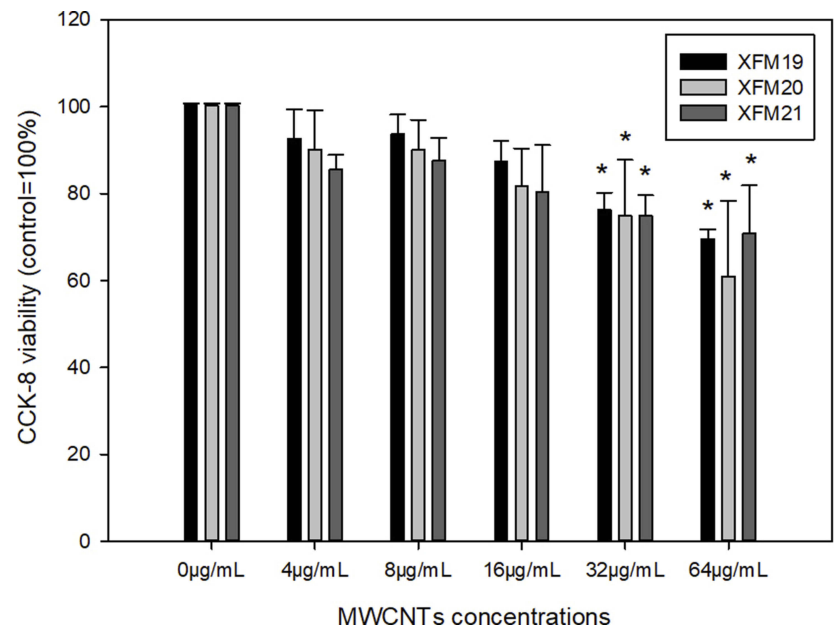

Figure 3 Cytotoxicity of MWCNTs to HUVECs. HUVECs were exposed to various concentrations of pristine MWCNTs (XFMI9), hydroxylated MWCNTs (XFM20) or carboxylated MWCNTs (XFM2I) for $24 \mathrm{hrs,} \mathrm{and} \mathrm{CCK-8} \mathrm{assay} \mathrm{was}$ done to indicate cytotoxicity. ${ }^{*} \mathrm{p}<0.0 \mathrm{I}$, compared with control.

groups (Figure 2), their physicochemical properties were still very similar (Figure 1 and Table 1), which might lead to similar cellular uptake efficiency of MWCNT into HUVECs (Figure 4 and Figure S2).

Previous studies showed that MWCNT exposure could promote oxidative stress. ${ }^{18,27,28}$ Moreover, Guo et al found that antioxidant could significantly alleviate the cytotoxicity and genotoxicity of MWCNTs to HUVECs, which further confirmed the role of oxidative stress in the cytotoxicity of MWCNTs. ${ }^{29}$ In the present study, we found that MWCNTs significantly increased intracellular ROS and decreased GSH (Figure 5), which suggested that MWCNTs could induce oxidative stress. However, it should be noticed that pristine MWCNTs, hydroxylated MWCNTs and carboxylated MWCNTs induced oxidative stress to a similar extent. Given the importance of oxidative stress in mediating the toxicological responses of MWCNTs, ${ }^{18}$ hydroxylation or carboxylation of MWCNTs does not change the cytotoxicity of MWCNTs due to the negligible impact of surface modifications on the oxidative stress induced by MWCNTs.

To further investigate the changes of signaling pathways due to hydroxylation and carboxylation, we measured the genes and proteins associated with ER stress. Previous study showed that NMs could promote prolonged ER stress leading to the activation of apoptosis pathway. ${ }^{19}$ In this study, we found that XFM19, XFM20 and XFM21 induced at least one of the following ER stress biomarkers, namely DDIT3, p-chop and chop (Figure 7), which suggest that these MWCNTs could promote ER stress. These results are consistent with previous studies showing that MWCNTs could induce ER stress in both cultured human cells ${ }^{12,13}$ and Caenorhabditis elegans. ${ }^{30}$ Meanwhile, the results from this study also showed that all types of MWCNTs significantly down-regulated the prosurvival ER stress gene XBP-1s. These effects could all contribute to the decrease of anti-apoptotic protein BCL-2 and induction of cleaved caspase-3/caspase-8 (Figure 6), finally contributing to the decrease of cellular viability. In mammalian cells, ER stress is sensed by three major ER-resident
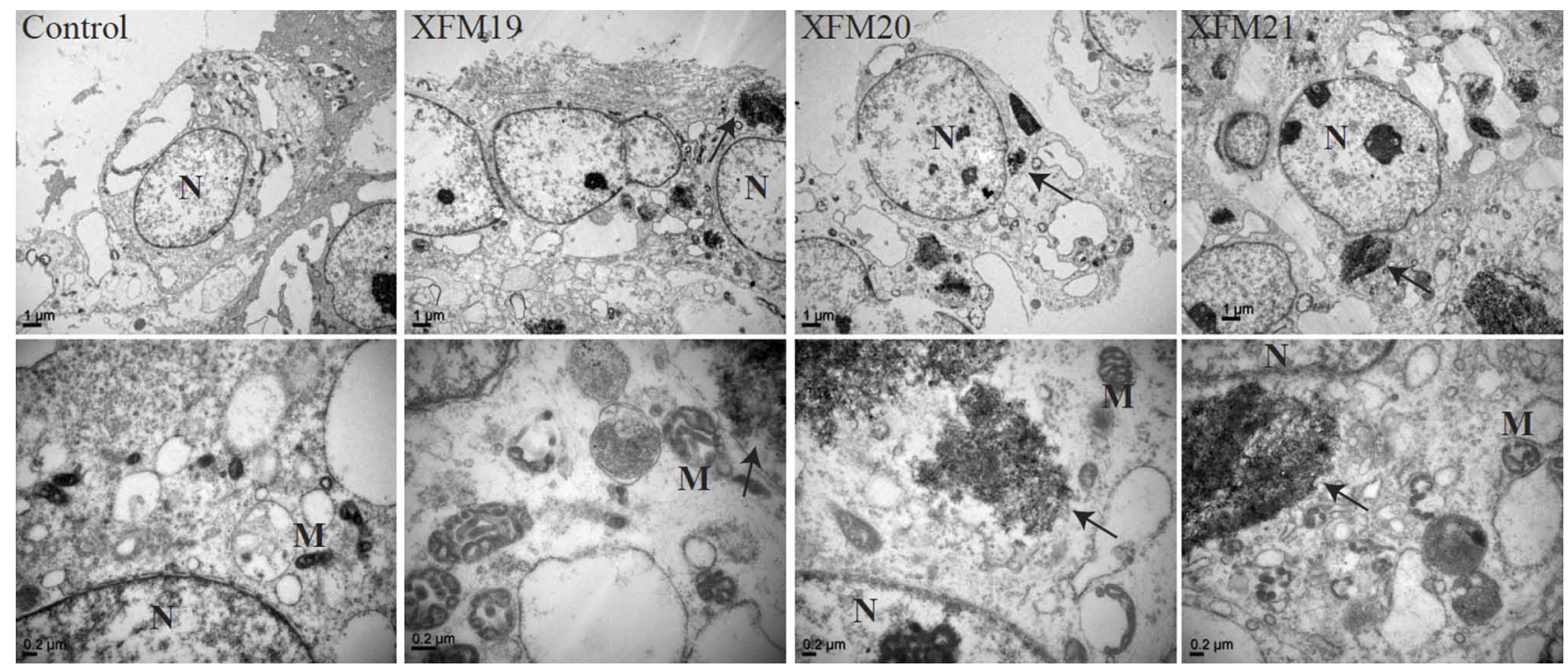

Figure 4 The ultrastructural changes of HUVECs. HUVECs were exposed to $64 \mu \mathrm{g} / \mathrm{mL}$ of pristine MWCNTs (XFMI9), hydroxylated MWCNTs (XFM20) or carboxylated MWCNTs (XFM2I). After 3 hrs exposure, TEM was used to image the ultrastructural changes of HUVECs and the internalized MWCNTs. The images in upper panel are images with low magnification, and the images in the lower panel are images with high magnification. The internalized MWCNTs were marked with arrows. Nuclei were marked with $\mathrm{N}$ and Mitochondria were marked with $\mathrm{M}$. 

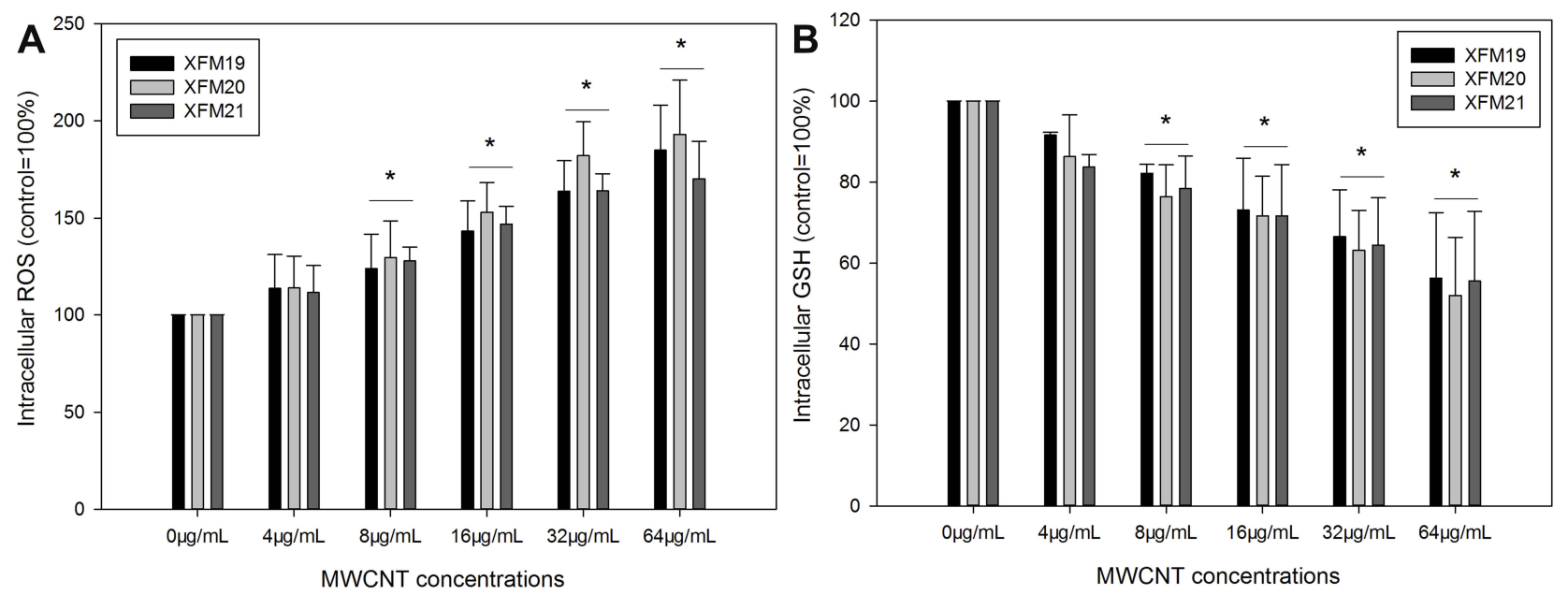

Figure 5 The changes of intracellular ROS (A) or GSH (B). HUVECs were exposed to various concentrations of pristine MWCNTs (XFMI9), hydroxylated MWCNTs (XFM20) or carboxylated MWCNTs (XFM2I) for $24 \mathrm{hrs,} \mathrm{and} \mathrm{intracellular} \mathrm{ROS} \mathrm{and} \mathrm{GSH} \mathrm{were} \mathrm{determined} \mathrm{by} \mathrm{using} \mathrm{a} \mathrm{fluorescent} \mathrm{probe.} \mathrm{*P<0.0I,} \mathrm{compared} \mathrm{with} \mathrm{control.}$

transmembrane molecules, namely IRE1 $\alpha$, protein kinase RNA-like ER kinase (PERK) and activating transcription factor-6 (ATF6). ${ }^{31}$ Moreover, we found that IRE1 $\alpha$ could be activated by XFM19 and XFM20 but not XFM21. It has been suggested that oxidative stress is closely related with NMinduced ER stress. ${ }^{19,20}$ A recent study by using systems toxicology approach revealed a cross talk between oxidative stress and ER stress in MWCNT-exposed Caenorhabditis elegans. ${ }^{30}$ Interestingly, we recently showed that antioxidants partially alleviated MWCNT-induced lipid accumulation through the modulation of ER stress, ${ }^{25,32}$ which further suggested a linkage between MWCNT-induced oxidative stress and ER stress. Here in this study, the data showed that all types of MWCNTs induced oxidative stress as well as ER stress.

One limitation for our study is that XFM20 and XFM21 were only modified with a low level of hydroxyl groups and carboxyl groups, respectively. However, increasing hydroxyl groups or carboxyl groups in MWCNTs with a diameter of about $30 \mathrm{~nm}$ is challenging, according to the supplier. It remains unclear if modification of MWCNTs with higher levels of hydroxyl groups or carboxyl groups could alter the biocompatibility of MWCNTs. Another limitation is

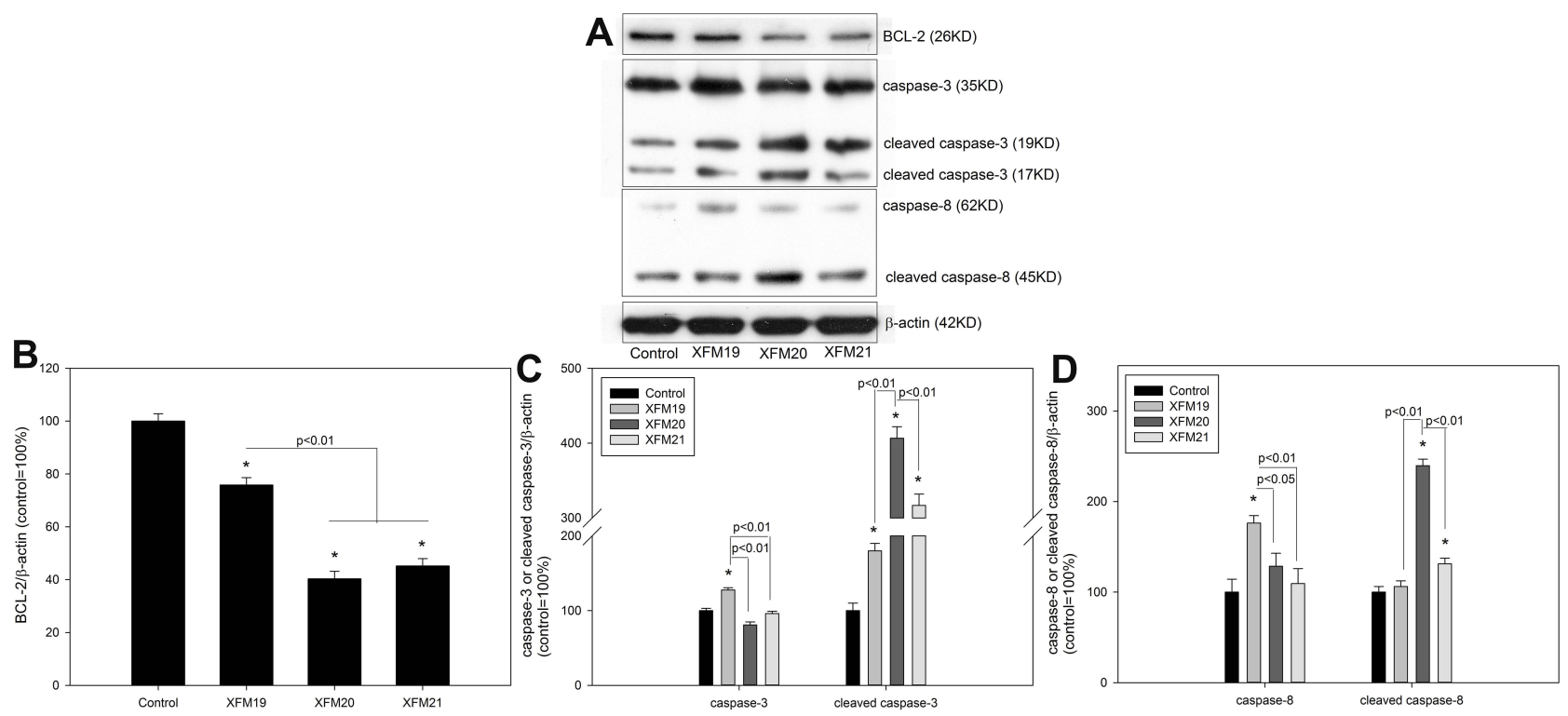

Figure 6 The protein levels of apoptotic proteins. HUVECs were exposed to $64 \mu \mathrm{g} / \mathrm{mL}$ of pristine MWCNTs (XFMI9), hydroxylated MWCNTs (XFM20) or carboxylated MWCNTs (XFM2I) for $24 \mathrm{hrs}$. After exposure, the levels of apoptotic proteins were measured by Western blot. (A) The Western blot bands; (B) the protein level of BCL2; (C) the protein level of caspase-3; (D) the protein level of caspase-8. ${ }^{*} \mathrm{p}<0.0 \mathrm{I}$, compared with control. 

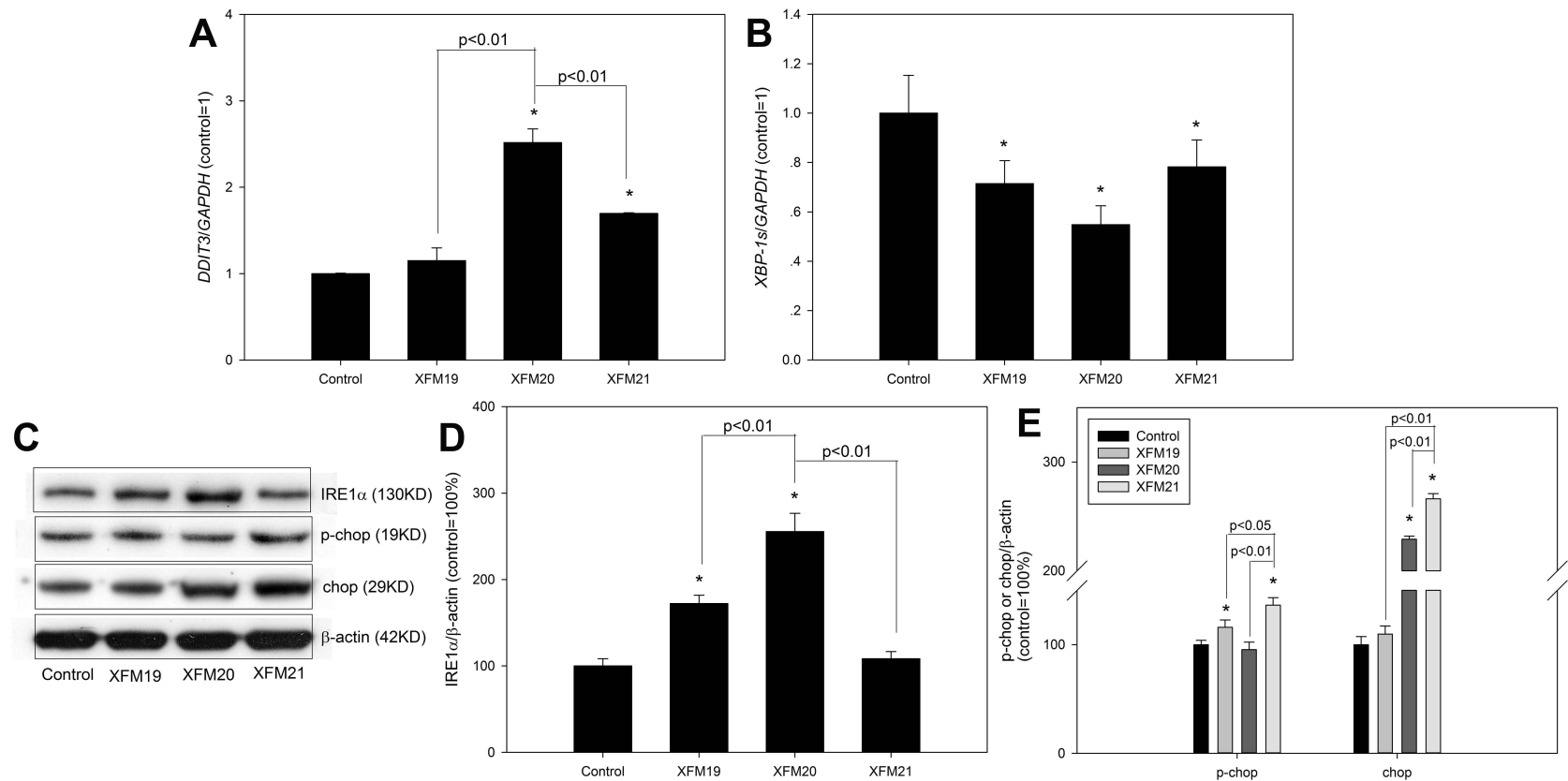

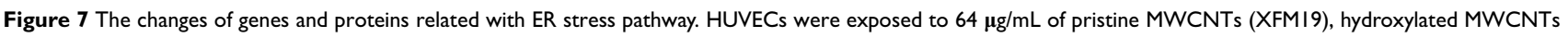
(XFM20) or carboxylated MWCNTs (XFM2I) for 24 hrs. After exposure, the expression of genes was determined by qRT-PCR, and protein level was measured by Western blot. (A) The expression of DDIT3; (B) the expression of XBP-Is; (C) the Western blot bands; (D) the protein level of IREI $\boldsymbol{\alpha}$; (E) the protein level of p-chop and chop. $*_{p}<0.05$, compared with control.

that this is a cell-based study, and in the future in vivo studies may be needed to confirm the influence of surface chemistry on biocompatibility of MWCNTs.

In summary, this study suggested that pristine, hydroxylated and carboxylated MWCNTs were equally cytotoxic to HUVECs. On the basis of the mechanism analysis, all types of MWCNTs induced oxidative stress and activated apoptosisER stress signaling pathway. Thus, surface modifications with a low degree of hydroxyl or carboxyl groups might not improve the biocompatibility of MWCNTs to human endothelial cells. As such, hydroxylated/carboxylated MWCNTs might need to be further surface-modified to improve their biocompatibility to human endothelial cells. ${ }^{5,33}$

\section{Funding}

We declare no financial support for this study.

\section{Disclosure}

No potential conflict of interest was reported by the authors.

\section{References}

1. De Volder MF, Tawfick SH, Baughman RH, Hart AJ. Carbon nanotubes: present and future commercial applications. Science. 2013;339:535-539. doi:10.1126/science.1222453

2. Liu L, Niu Z, Chen J. Flexible supercapacitors based on carbon nanotubes. Chinese Chem Lett. 2018;29:571-581. doi:10.1016/j.cclet.2018.01.013
3. Yang Y, Chen C, Hu J, Deng Y, Zhang Y, Yang D. High performance lithium-sulfur batteries by facilely coating a conductive carbon nanotube or graphene layer. Chinese Chem Lett. 2018;29:1777-1780.

4. Hemasa AL, Naumovski N, Maher WA, Ghanem A. Application of carbon nanotubes in chiral and achiral separations of pharmaceuticals, biologics and chemicals. Nanomaterials (Basel). 2017;7:E186. doi: $10.3390 /$ nano 7070186

5. Saleem J, Wang L, Chen C. Carbon-based nanomaterials for cancer therapy via targeting tumor microenvironment. Adv Healthcare Mater. 2018;7:e1800525. doi:10.1002/adhm.v7.20

6. Maas M. Carbon nanomaterials as antibacterial colloids. Materials (Basel). 2016;9:ma9080617. doi:10.3390/ma9080617

7. Cao Y, Gong Y, Liu L, et al. The use of human umbilical vein endothelial cells (HUVECs) as an in vitro model to assess the toxicity of nanoparticles to endothelium: a review. $J$ Appl Toxicol. 2017;37:1359-1369. doi:10.1002/jat.v37.12

8. Setyawati MI, Tay CY, Docter D, Stauber RH, Leong DT. Understanding and exploiting nanoparticles' intimacy with the blood vessel and blood. Chem Soc Rev. 2015;44:8174-8199. doi: 10.1039/C5CS00499C

9. Ajdary M, Moosavi MA, Rahmati M, et al. Health concerns of various nanoparticles: a review of their in vitro and in vivo toxicity. Nanomaterials (Basel). 2018;8:nano8090634. doi:10.3390/nano 8090634

10. Deng C, Hao T, Li Z, Fan Q. Physicochemical properties that determine cellular transport of nanocarriers in vitro and in vivo. Curr Org Chem. 2016;20:1835-1848.

11. Alshehri R, Ilyas AM, Hasan A, Arnaout A, Ahmed F, Memic A. Carbon nanotubes in biomedical applications: factors, mechanisms, and remedies of toxicity. $J$ Med Chem. 2016;59:8149-8167. doi:10.1021/acs.jmedchem.5b01770

12. Long J, Xiao Y, Liu L, Cao Y. The adverse vascular effects of multi-walled carbon nanotubes (MWCNTs) to human vein endothelial cells (HUVECs) in vitro: role of length of MWCNTs. J Nanobiotechnology. 2017;15:80. doi:10.1186/s12951-017-0318-x 
13. Zhao X, Chang S, Long J, Li J, Li X, Cao Y. The toxicity of multi-walled carbon nanotubes (MWCNTs) to human endothelial cells: the influence of diameters of MWCNTs. Food Chem Toxico. 2019;126:169-177. doi:10.1016/j.fct.2019.02.026

14. Hamilton RF, Wu Z, Mitra S, Holian A. The effects of varying degree of MWCNT carboxylation on bioactivity in various in vivo and in vitro exposure models. Int J Mol Sci. 2018;19:E354. doi:10.3390/ijms 19020354

15. Liu Z, Dong X, Song L, et al. Carboxylation of multiwalled carbon nanotube enhanced its biocompatibility with L02 cells through decreased activation of mitochondrial apoptotic pathway. $J$ Biomed Mater Res A. 2014;102:665-673. doi:10.1002/jbm.a.v102.3

16. De Marchi L, Neto V, Pretti C, et al. Toxic effects of multi-walled carbon nanotubes on bivalves: comparison between functionalized and nonfunctionalized nanoparticles. Sci Total Environ. 2018;622-623:1532-1542. doi:10.1016/j.scitotenv.2017.10.031

17. Orecna M, De Paoli SH, Janouskova O, et al. Toxicity of carboxylated carbon nanotubes in endothelial cells is attenuated by stimulation of the autophagic flux with the release of nanomaterial in autophagic vesicles. Nanomedicine. 2014;10:939-948. doi:10.1016/j.nano.2014.02.001

18. Moller P, Christophersen DV, Jensen DM, et al. Role of oxidative stress in carbon nanotube-generated health effects. Arch Toxicol. 2014;88:1939-1964.

19. Cao Y, Long J, Liu L, et al. A review of endoplasmic reticulum (ER) stress and nanoparticle (NP) exposure. Life Sci. 2017;186:33-42. doi:10.1016/j.lfs.2017.08.003

20. Liu N, Tang M. Toxic effects and involved molecular pathways of nanoparticles on cells and subcellular organelles. J Appl Toxicol. 2019. doi: $10.1002 /$ jat.3817

21. Bhattacharjee S. DLS and zeta potential - what they are and what they are not? J Control Release. 2016;235:337-351. doi:10.1016/j. jconrel.2016.06.017

22. Tucureanu V, Matei A, Avram AM. FTIR spectroscopy for carbon family study. Crit Rev Anal Chem. 2016;46:502-520. doi:10.1080/ 10408347.2016.1157013

23. Gutierrez-Hernandez JM, Ramirez-Lee MA, Rosas-Hernandez H, et al. Single-walled carbon nanotubes (SWCNTs) induce vasodilation in isolated rat aortic rings. Toxicol in Vitro. 2015;29:657-662. doi:10.1016/j.tiv.2015.02.001
24. Chang S, Zhao X, Li S, et al. Cytotoxicity, cytokine release and ER stress-autophagy gene expression in endothelial cells and alveolar-endothelial co-culture exposed to pristine and carboxylated multi-walled carbon nanotubes. Ecotoxicol Environ Saf. 2018;161:569-577. doi:10.1016/j.ecoenv.2018.06.025

25. Yang H, Li J, Yang C, Liu H, Cao Y. Multi-walled carbon nanotubes promoted lipid accumulation in human aortic smooth muscle cells. Toxicol Appl Pharmacol. 2019;374:11-19. doi:10.1016/j.taap.2019. 04.022

26. Zhao C, Zhou Y, Liu L, et al. Lipid accumulation in multi-walled carbon nanotube-exposed HepG2 cells: possible role of lipophagy pathway. Food Chem Toxicol. 2018;121:65-71. doi:10.1016/j. fct.2018.08.033

27. Abu GM, El-Magd MA. Effect of pristine and functionalized multiwalled carbon nanotubes on rat renal cortex. Acta Histochem. 2019;121:207-217. doi:10.1016/j.acthis.2018.12.005

28. Yu X, Hong F, Zhang YQ. Bio-effect of nanoparticles in the cardiovascular system. J Biomed Mater Res A. 2016;104:2881-2897. doi:10.1002/jbm.a.35804

29. Guo YY, Zhang J, Zheng YF, Yang J, Zhu XQ. Cytotoxic and genotoxic effects of multi-wall carbon nanotubes on human umbilical vein endothelial cells in vitro. Mutat Res. 2011;721:184-191. doi:10.1016/j.mrgentox.2011.01.014

30. Eom HJ, Roca CP, Roh JY, et al. A systems toxicology approach on the mechanism of uptake and toxicity of MWCNT in Caenorhabditis elegans. Chem Biol Interact. 2015;239:153-163. doi:10.1016/j.cbi. 2015.06.031

31. Ozcan L, Tabas I. Role of endoplasmic reticulum stress in metabolic disease and other disorders. Annu Rev Med. 2012;63:317-328. doi:10.1146/annurev-med-043010-144749

32. Long J, Ma W, Yu Z, Liu H, Cao Y. Multi-walled carbon nanotubes (MWCNTs) promoted lipid accumulation in THP-1 macrophages through modulation of endoplasmic reticulum (ER) stress. Nanotoxicology. 2019;13:938-951. doi:10.1080/17435390.2019.159 7204

33. Gao S, Tang G, Hua D, et al. Stimuli-responsive bio-based polymeric systems and their applications. J Mater Chem B. 2019;7:709-729. doi:10.1039/C8TB02491J
International Journal of Nanomedicine

\section{Publish your work in this journal}

The International Journal of Nanomedicine is an international, peerreviewed journal focusing on the application of nanotechnology in diagnostics, therapeutics, and drug delivery systems throughout the biomedical field. This journal is indexed on PubMed Central, MedLine, CAS, SciSearch ${ }^{\mathbb{R}}$, Current Contents ${ }^{\mathbb{R}} /$ Clinical Medicine, $^{2}$

\section{Dovepress}

Journal Citation Reports/Science Edition, EMBase, Scopus and the Elsevier Bibliographic databases. The manuscript management system is completely online and includes a very quick and fair peer-review system, which is all easy to use. Visit http://www.dovepress.com/ testimonials.php to read real quotes from published authors. 